\title{
TROIS REPERES DANS LA CONSTRUCTION DU PROJET ADOLESCENT: HISTOIRE PERSONNELLE; CULTURE; ASSOCIATIONS
}

\author{
THREE LANDMARKS IN THE CONSTRUCTION OF THE \\ ADOLESCENT PROJECT: PERSONAL HISTORY; \\ CULTURE; ASSOCIATIONS
}

Emile-Henri Riard ${ }^{1, *}$

\begin{abstract}
RESUMÉ: La dimension imaginaire, les situations multiculturelles tout comme le rôle des associations de quartier sont souvent négligées dans les recherches portant sur la construction du projet des adolescents. Cet article vise à en dégager l'impact auprès de jeunes vivant en France, issus de milieux culturellement différenciés. Le cadre théorique est celui de BLOS et MAHLER (séparation/individuation), complété par les apports de KAËS sur les concepts de transitionnalité (1979), de filiation/ affiliation (1985); d'idéologie (2016) qui ressortent de façon récurrente de recherches menées depuis 1997.
\end{abstract}

Mots-clés: Projet adolescent. Culture. Associations. Instances psychiques. Transitionnalité et filiation.

ABSTRACT: The imaginary dimension, multicultural situations and the role of neighbourhood associations are often overlooked in research on the construction of the adolescent project. This article aims to identify the impact on young people living in France, from culturally differentiated backgrounds. The theoretical framework is that of BLOS and MAHLER (separation/individuation), supplemented by KAËS' contributions on the concepts of transitionality (1979), filiation/affiliation (1985); ideology (2016), which have been the result of searches conducted since 1997.

Keywords: Adolescent project. Culture. Associations. Psychic agency. Transitionality and filiation.

1.Université Picardie Jules Verne - Laboratório CAREF - Amiens, France.

*Auteur correspondant: eh.riard@laposte.net

Dossier organisé par: Joyce Mary Adam e Débora Cristina Fonseca 


\section{Introduction}

L'adolescence est, pour les jeunes qui en sont l'objet, le temps des remaniements psychiques profonds, décisifs, les adolescents devant abandonner les identifications de l'enfance et se tourner vers le futur pour accéder à un temps personnel, celui de l'identité. C'est à ce moment que se pose à eux la question du projet «naturellement » orienté vers le futur, des contenus de ce dernier et des moyens nécessaires pour y parvenir. Ce vecteur leur permettra d'accéder au statut adulte et, par voie de conséquence, à leur future insertion sociale et professionnelle. Il concerne plus spécifiquement, associés à un jeu de valeurs, les deux éléments indissociables de tout fonctionnement humain: celui de la réalité et celui de l'imaginaire, ce dernier étant le plus souvent absent ou pour le moins négligé dans les approches que la société a du projet, notamment l'école. En outre, cette construction, tributaire des changements adolescents de tous ordres (somatiques, psychiques, sociaux), s'effectue toujours à un moment particulier de l'histoire de la famille du jeune et de son groupe d'appartenance, dans une aire culturelle donnée, situation qui n'est pas sans impact sur cette construction.

L’objectif de cet article est de présenter trois éléments souvent négligés dans les recherches concernant le projet adolescent qui n'en jouent cependant pas moins un rôle fondamental dans sa construction, afin d'en analyser l'impact sur celle-ci. 1 - L'histoire personnelle du sujet, en orientant le focus sur la dimension imaginaire et ses rapports avec la réalité. 2 - La culture, dont l'intérêt tient surtout à la place de l'héritage reçu, essentiellement des parents des jeunes (héritage de comportements, de valeurs, de codes) qui posent la question de la filiation et de l'affiliation et celle des difficultés lorsque le code culturel (parental) reçu ne correspond pas ou seulement partiellement avec celui de la société de résidence du jeune. 3 - Enfin, les associations de quartier qui, fréquentées par les jeunes sur la base du volontariat, leurs offrent, en complément de la famille et de l'école, des possibilités de réussir la construction de leur projet alors qu'ils rencontraient des difficultés qui ne pouvaient être traitées positivement par aucune de ces deux instances.

Les contenus de cet article sont issus de travaux développés dans le cadre de recherches ayant fait l'objet de contrats associant différents types de partenaires ${ }^{1}$. Les équipes étaient constituées de psychologues (principalement) et de sociologues. Ces travaux sont également issus de doctorats en psychologie sociale clinique réalisés sous la direction de l'auteur de cet article (MAINE, 2009; HOANG, 2012). Ces travaux ont été menés dans des milieux socialement et culturellement diversifiés, essentiellement en France et au Maroc, sur la base d'échantillons représentatifs. Le nombre total de sujets pris en compte dans cet article est de 650. Les sujets ont toujours été répartis de façon sensiblement égale entre: - filles et garçons; selon l'âge: 12-13 ans 11 mois, 14-15 ans 11 mois, 16 à 18-20 ans; et selon le milieu de résidence (rural ou urbain) lorsque cela était nécessaire².

Outre le questionnaire adapté à chaque recherche qui a porté sur le projet du jeune selon donc la dimension de la réalité, une épreuve à orientation projective a été systématiquement proposée, ayant pour fonction de mettre à jour les mécanismes de l'imaginaire (mécanismes de défense spécifiques, représentations, mode de résolution des conflits...). Elle a consisté à demander aux sujets d'imaginer une histoire à partir de cartes projectives issues du Thematic Apperception Test de Murray (1953) qui leur étaient proposées. La consigne retenue a été celle proposée par MURRAY3.

Après un très bref rappel des procédures suivies par l'école dans les mesures d'aide à la construction du projet des jeunes qu'elle leurs propose, qui consiste à appliquer une démarche issue des directives du Ministère de l'Education Nationale, sera abordée la place qu'occupe l'histoire personnelle du sujet dans cette construction via les remaniements spécifiques de cette période 
de la vie (1). Elle sera suivie des retentissements particuliers que l'histoire personnelle peut avoir pour ceux des jeunes issus d'une autre culture, notamment dans leur choix de vie, posant de facto la question des rapports qu'entretiennent filiation et affiliation (2). Enfin la dernière partie proposera une piste de réponse possible pour ceux des jeunes en difficulté de projet : celle procurée par les associations $(3)^{4}$.

\section{École et Construction du Projet}

Une des tâches essentielles que l'école et les familles demandent aux jeunes, en France, est de construire un projet, le plus souvent considéré sous sa seule dimension professionnelle. A cet effet, l'école met en place un dispositif allant de la classe de sixième à celle de troisième (de 11-12 ans à 14-15ans). D’abord fondé sur une réflexion proposée aux élèves sur leur avenir, ce travail auprès des jeunes s'appuie ensuite sur des tests et sur des entretiens réalisés avec un Conseiller d'Orientation Psychologue (C.O.P.). Au terme de la quatrième année de collège, les jeunes intègrent soit un lycée technique et professionnel, soit un lycée d’enseignement général. En fonction de leurs résultats scolaires, certains sont cependant orientés plus tôt.

La construction du projet professionnel est ainsi conçue comme s'effectuant sur des choix préalables et successifs, en appui sur des facteurs individuels incluant les désirs des jeunes. La démarche suivie par l'école s'inspire des travaux scientifiques qui renvoient aux trois conceptions bien connues, développées successivement par GINZBERG et al. (1951); CRITES (1962) et SUPPER (1988). Le premier met l'accent sur le développement dit « vocationnel », le choix professionnel de l'adolescent résultant alors d'un processus irréversible constitué d'abord de choix dits «fantaisistes » qui deviennent de plus en plus « réalistes ». CRITES (1962) puis SUPPER (1988) introduisent l'idée de « maturité professionnelle » qui repose sur une mobilisation de l'individu dans son cheminement vers une solution qui prend en compte ses aptitudes, ses motivations..., et une cristallisation progressive sur ses intérêts et sur les valeurs de travail, dans la recherche d'une cohérence entre les préférences professionnelles et l'estime de soi. HUTEAU (1993) élargi cette question en la plaçant sur le terrain des représentations, essentiellement familiales et identitaires, reliant ainsi profession et identité, établissant un rapport entre représentation de soi et représentations professionnelles (celles-ci étant surtout issues de la famille). GUICHARD (1993) va dans le sens d'une influence déterminante des représentations que les adolescents ont de l'activité professionnelle de leurs parents sur leur projet.

Cependant, une autre approche, qui ne remet en question ni les approches antérieures, ni celles développées ultérieurement, a été esquissée par MÂLE (1962), psychiatre, pour qui les choix professionnels ont des origines archaïques et œdipiennes et prennent donc également racine dans l'imaginaire des jeunes.

Partant de l'ensemble des travaux précités et se plaçant dans une perspective intégrative, il a été proposé de considérer le projet professionnel non pas comme un élément isolé du reste de la vie et dépendant des seuls éléments spécifiques classiquement avancés, les intérêts, attitudes, compétences, etc... , mais comme une partie fondamentale d'un ensemble, d'un «tout ", constitué par la personne du jeune, renvoyant à l'idée d'un projet unique constitué de facettes dont les deux principales sont celles de vie professionnelle et de vie privée. Il rassemble et place en interdépendance les dimensions affective, cognitive, intellectuelle et sociale de l'individu en lien intime avec son développement. La définition qui en a été proposée est la suivante : «le projet est un acte d’engagement du sujet dans l'avenir, un fil directeur plaçant en interdépendance les dimensions manifestes et latentes, les dimensions affectives 
et cognitives, le passé avec le présent et le futur »(RIARD, 2004, p. 18). Les travaux de CASTELLAN et RIARD (2005) et de CASTELLAN (2007), confortent cette approche qui constitue la base de cet article.

\title{
Histoire Personnelle du Sujet
}

\author{
Une situation et contexte théorique
}

Lapproche du développement psychique proposée par les psychanalystes MAHLER (1982) et BLOS (1967), retenue ici et adoptée dans la plupart des travaux scientifiques, est connue sous le nom de " processus de séparation/individuation ». Le développement y est conçu comme se déroulant en deux étapes majeures. La première concerne surtout la petite enfance et consiste essentiellement en un travail de séparation (surtout physique) avec la mère, renfermant les bourgeons de l'individuation. Ce travail se poursuit à bas bruit au cours de la période de latence (dominée quant à elle par les dimensions cognitive, intellectuelle et sociale). La seconde étape, également étalée sur plusieurs années est « organisée » en quelque sorte par l'individuation, par la construction identitaire. Elle est déclenchée par une montée puissante et brutale des affects qui accompagne la résurgence du complexe d'CEdipe et ramène en surface de nombreux matériaux psychiques, tels des représentations, des mécanismes de défense, des fantasmes... parfois très anciens de l'histoire du sujet, le plus souvent revus et corrigés par son histoire plus récente. Elle lui permet de prendre ses distances avec le monde de l'enfance, essentiellement avec les figures parentales et leurs représentants, le jeune abandonnant, tout au moins devant le faire, la dépendance infantile vs la toute-puissance parentale (présentes à la période de latence). C'est donc dans un contexte de réorganisations multiples et profondes des relations objectales que se poursuit le travail de construction du projet qui doit permettre au jeune, à terme, d'accéder et d'assumer les deux fonctions majeures de l'état adulte : celle de "producteur ", qui concerne le monde social et en particulier professionnel, et celle de "reproducteur » relatif à la vie affective. Ces deux fonctions devront en outre être assorties d'un jeu de valeurs.

Voyons maintenant en quoi consiste le retraitement des matériaux psychiques localisés au cœur du processus d'adolescence. Quels en sont les contenus et leurs relations, tant entre eux qu'avec la dimension manifeste du projet?

Matériaux et mécanismes impliqués dans la construction du projet

La littérature d’obédience psychanalytique consacrée au processus d'adolescence est particulièrement féconde et convergente quant à la présence des matériaux qui y sont présents et impliqués (KESTEMBERG, 1962; BLOS, 1967; ERIKSON, 1968; GUTTON, 1983; CHABERT, 1993; JEAMMET, 1983; MARCELLI ; BRACONNIER, 2007; LESOURD, 2005; GOLSE, 2008). Les remaniements adolescents y sont présentés comme concernant essentiellement trois instances psychiques qui ont pour particularité d’être toutes trois présentes d'un bout à l'autre de la vie, assurant ainsi la continuité du sujet en dépit de ses transformations. Ce sont: les identifications (qui concernent essentiellement le passé infantile et ciblent les parents en particulier); les idéaux du moi (qui sont l'expression de désirs personnels orientés vers le futur et dont l'apparition est corrélative de la baisse du surmoi - qui est quant à lui d’origine parentale). Enfin, la balance narcissico-objectale, qui connaît à cette période de la vie une redistribution des rapports entre les dimensions objectales et narcissiques (c'est-à-dire entre les places respectives faites à l'autre et à soi - en particulier à l'autre sexe - dans le fonctionnement psychique 
des jeunes). Sa présence est fondamentale dans la recherche d'un équilibre fondé désormais sur la complémentarité et non plus sur la simple différence des sexes, complémentarité également nécessaire entre les dimensions cognitive et affective de ce qui est indispensable à l'équilibre de tout individu. Comment et sous quelle forme ces matériaux interviennent-ils dans la construction du projet adolescent?

Pour répondre à cette question, il est nécessaire de prendre des points de repère : ce sont les deux formes majeures de projet telles qu'identifiées à partir de résultats de travaux de recherche menés auprès de jeunes de 11-12 ans à 15-16 ans. Il s'agit du projet dit "bien engagé » et du " projet faible ", parfois «magique» (RIARD, 1996; RIARD; DACHMI, 2004).

Dans un projet «bien engagé », le jeune recherche activement des informations sur la profession qu'il projette, recourt à des aides, se documente, abandonne rationnellement ses projets antérieurs, jette un regard sur lui-même et sur ses capacités, sur ses aptitudes, prend également en compte ses centres d'intérêt tout comme il accorde une place aux autres (relations amicales, voire amoureuses), et il se projette à une dizaine d'années, adoptant également un jeu de valeurs.

Inversement, le projet «faible » se traduit essentiellement par des éléments épars, est souvent ramené à la seule dimension affective, et son auteur néglige toute recherche d'informations sur les professions, est en difficulté à se projeter dans le futur, ne s'interrogeant par ailleurs pas ou peu sur ses intérêts et ses capacités.

\section{Caractéristiques de l'engagement du jeune dans son projet}

\section{Présence de correspondances entre les dimensions manifeste et imaginaire du projet}

Des résultats de travaux croisant entretiens et épreuves de nature projective de type Thematic Apperception Test (T.A.T.), constitué de cartes projectives (cf.note2), p.e.MAINE,2009; CASTELLAN, 2007; RIARD, 1996; RIARD; DACHMI, 2004, il ressort la présence de correspondances entre la qualité du projet telle qu'observée au niveau manifeste et celle relevée au niveau latent. Par exemple, à un projet dit « bien engagé » (ci-dessus), correspond, à un niveau latent, un travail de réorganisation des relations objectales orienté vers l'accès à l'état adulte (retraitement des identifications de l'enfance - présence d'un travail de séparation et de conflictualisation vis-à-vis des figures parentales; présence d'idéaux plus personnels; place importante accordée à l'autre sexe dans des relations s'inscrivant dans des rapports de complémentarité et non plus de simple différence). Inversement, à un projet manifeste «faible» (ci-dessus) correspond au niveau latent une faiblesse d'orientation du travail psychique menant à létat adulte, ce qui maintient encore leurs auteurs dans un statut à coloration infantile. Ce qui se traduit par un travail de séparation et de conflictualisation faiblement engagé, voire inexistant; par la mise en avant de peu de désirs personnels et par une place considérable, voire outrancière, accordée aux relations de nature narcissique, l'autre - en particulier l'autre sexe - n’«existant» pas, si ce n'est, en quelque sorte, pour «tenir le miroir», c'est-à-dire pour renvoyer au sujet sa propre image.

\section{Caractère synchrone de la qualité des composantes des instances psychiques}

Comme on l'aura relevé, il ressort de la caractéristique précédente que le travail réalisé au niveau de chacune des trois instances va toujours dans le même sens au même moment. Il en est ainsi des composantes observées dans le travail sous-tendant le projet «bien engagé » qui présentent toutes de qualités comparables au même moment : présence de la séparation du conflit avec les figures parentales, ET mise en place d'idéaux personnels, ET représentations de l'autre sexe sous l’angle de la complémentarité. 
Les trois instances issues de l'imaginaire sont également de qualité comparable, au même moment lorsque le projet du jeune est «faible»: impossibilité ou faiblesse de la (nécessaire) conflictualisation ou de la séparation avec les figures parentales; absence d'idéaux personnels; grande difficulté voire incapacité à s'envisager dans des relations de l'ordre de la complémentarité avec l'autre sexe qui n'existe alors en quelque sorte que pour «tenir le miroir».

Cette synchronicité des matériaux de l'imaginaire renvoie à la question du mode de fonctionnement psychique.

\section{Construction du projet sur un mode de nature oscillatoire}

L’analyse des résultats de cette construction conduit dans un premier temps à poser comme hypothèse que les composantes issues des trois instances impliquées peuvent être regroupées en pôles de relations objectales sur la base de leurs points communs en termes de distance relationnelle. Dans l'un des pôles, la qualité des matériaux place l'adolescent dans un jeu de relations fondées sur la recherche de la différence / complémentarité (spécifiques de l’état adulte). Les relations y sont caractérisées par un travail de retraitement des identifications de l'enfance; par la mise en place d'idéaux personnels, et par une redistribution des rapports entre les dimensions narcissiques et objectales dans le sens de la recherche d'une complémentarité avec l'autre sexe. Lautre pôle est quant à lui constitué de matériaux spécifiant des relations de l'ordre de la proximité /similitude, concernant donc des modes de relations inhérents à l'état infantile - ou proche de ce dernier - dans la mesure où le travail qui y est accompli est faible en direction du statut adulte. Ces relations contribuent à maintenir le sujet dans la dépendance infantile $v s$ la toute-puissance parentale ; l'autre sexe y est encore vu sous l'angle de la simple différence, de même que les désirs personnels sont inexistants ou d’ordre " magique ».

Dans l'immense majorité des cas, les adolescents construisent leur projet en dépit des difficultés qu'ils rencontrent, ce qui implique de leur part d'avoir à traiter des relations objectales afin, progressivement, d’abandonner la position infantile. Or, pour tous les jeunes, on observe généralement des moments de « marche en avant » du projet vers le statut adulte (temps de progression), suivis à d'autres moments d'un retrait, d'un changement, d'un arrêt plus ou moins durable de ce projet (temps de retrait). On peut en déduire la présence d'un mouvement de nature oscillatoire entre les deux pôles de relations précitées, alternant des mouvements "progrédients » et « régrédients ». Ce mouvement est explicable par la présence de l'angoisse du futur et de ses incertitudes, mais aussi, au niveau des relations, par ce qui est vécu comme une trop grande proximité avec l'« autre ", par exemple avec l'autre sexe, proximité qui peut être perçue et vécue comme insoutenable dans un premier temps car le sujet n'est pas capable d'en assumer les conséquences, c'est-à-dire, au moins d'envisager de modifier ses relations avec l'autre sexe en adoptant une nouvelle position relationnelle qui serait alors fondée sur des relations de complémentarité et non plus sur l'existence de simple différences (entre les sexes). D’un autre côté, ce mouvement peut être explicable par ce qui est vécu comme une trop grande distance avec l' " autre ", notamment parce que cette distance contribue à maintenir le jeune dans un état infantile, ce dont son corps témoigne de l'inadéquation, et ce qui lui est également insoutenable (CHABERT, 1993).

Ce qui signifie également que le travail de construction du projet n'est pas strictement linéaire, ce que pensent et appliquent dans leur démarche un certain nombre d'intervenants professionnels ou de parents. Ces mouvements oscillatoires permettent un travail d'intégration progressive de données portant sur les relations du jeune tant avec lui-même quavec les autres et avec son environnement social et culturel. 


\section{Culture(s) et Construction du Projet Adolescent}

Dans une société en mouvement constant, dans une mondialisation reposant sur les mouvements migratoires, mais aussi parce qu'il s'agit d'un enjeu sociétal et de cas fréquents, il est indispensable d'aborder la question de la construction du projet d'adolescents dont les parents ou grands-parents ont migré. Pour illustrer le propos, nous prendrons le cas de jeunes dont les ancêtres, Marocains, se sont établis en France.

\section{Une situation}

L'idée de projet est relativement nouvelle au Maroc (RIARD; DACHMI, 2004; RIARD, 2005), car jusquà une époque très récente, le fils recevait sa profession de son père qui la lui imposait. Il n’avait donc pas la possibilité d'exprimer ses propres désirs. C'est dans ce contexte que sont arrivés en France les parents ou grands-parents de ces jeunes. Si chez ces derniers le travail psychique de remaniements est sensiblement comparable à celui observé chez les autres adolescents, la construction de leur projet, dans la société dite d'accueil les place cependant en regard de difficultés supplémentaires dont certaines sont inhérentes à la teneur de leur héritage culturel, celui-ci introduisant des différences pouvant être vécues comme pénalisantes par rapport aux jeunes autochtones.

\section{L'héritage culturel et ses composantes}

Tous les jeunes doivent composer avec leur histoire et celle de leurs parents (ce qu'on leur en a transmis, ce qu'ils en ont retenu et en ont rejeté; CICCONE, 1997). C'est leur héritage, c'est-à-dire, selon KAËS (1979, p. 26), « ce qui relie l'individu au social, et (c'est) ce qui permet d’encoder et de décoder les représentations et les affects plus ou moins souplement organisés dans une aire culturelle donnée et pour un sujet singulier ». Or, l'héritage est composé de codes relatifs aux valeurs, aux façons d'investir les objets, aux modes de relations avec les autres, aux représentations de l'homme et de la femme, tout autant quà l'intérêt porté à l'école, ou au sens de l'honneur, à la place faite au temps, ou bien encore à la religion, à la tradition. Et toutes ces variables sont présentes à un titre ou à un autre dans le projet du jeune dont elles contribuent à en dessiner les contours et la qualité, outre le fait que leur observance permet d'assurer sa sécurité dans la culture qui les contient. Cet héritage étant nécessairement plus lourd et difficile à assumer pour les jeunes dont les parents ou grands-parents ont migré du fait de la multiplicité des références culturelles et, donc, de choix à réaliser (RIARD ; ALAGUI, 2013).

\section{Retentissements de la culture sur le projet}

\section{Retentissements pour le jeune}

Placés dans une aire culturelle différente de celle de leurs ancêtres, nombre de jeunes se ressentent comme étant en "porte-à-faux » avec tout ou partie de leur héritage puisque leur projet les contraint de faire un choix de codes ou de parties de code, c'est-à-dire, à choisir entre ceux proposés par la culture de leurs ancêtres et ceux de la culture du pays dit « d'accueil ». Nombre d'entre eux sont ainsi tiraillés entre leur fidélité à la culture de leurs ancêtres et ce qui découle de leur propre expérience acquise à lécole, dans des associations et auprès des médias du pays de vie.

Il en découle que certains de ces jeunes sont soumis à de fortes tensions génératrices d'un malêtre le plus souvent sourcé dans des conflits pour lesquels ils ne trouvent pas toujours d'issue. Ces tensions 
peuvent également être dues à une inversion souvent observée des représentations de leurs relations à l'autre sexe, comparativement à celles des jeunes européens ; ou encore à une pauvreté de l'idéal du moi, ce qui est repérable par la faiblesse de leur projection dans le futur, en particulier celle d'idéaux personnels. Enfin, elles peuvent également naître de la séparation très grande, parfois assimilable à du clivage, observé entre les univers familiaux et scolaires (RIARD; PEYRE, 1997; RIARD, 2011). Et lorsqu'il y a modification de code (cf. ci-dessous), ces difficultés se traduisent non seulement par un mal-être, mais elles génèrent également un sentiment d'insécurité, une culpabilité due au fait que ces modifications sont vécues comme des transgressions vis-à-vis de la culture de leurs origines, comme une infidélité à cette dernière.

\section{Retentissements sur l'entourage du jeune}

Le choix de codes opéré par les adolescents n’est pas sans retentissements sur leurs relations avec leurs parents et il soulève principalement la question des rapports entre filiation et affiliation (KAËS, 1985). Ainsi, lorsque les codes retenus par le jeune sont très proches de ceux des origines ancestrales, le jeune s'inscrit dans une filiation, dans une continuité entre générations, et le père du jeune est alors en accord avec sa communauté qui le reconnaît en retour comme un « bon père » (MEDEJEL, 1998). En revanche, lorsque le jeune fait choix de codes culturels éloignés de ceux de ses ancêtres, s’affiliant ainsi à d’autres valeurs, lorsqu'il y a modifications importantes de code entre deux générations, le jeune est non seulement lui-même en « danger » dans ses relations avec sa famille, mais cette dernière l'est également, car le père ne peut plus alors penser son fils sur la base d'une continuité culturelle, encourant par là-même lui-même le risque de ne plus être reconnu comme un «bon père » par sa communauté du fait qu’il n’a pas été « capable » d’y maintenir son fils.

Certains de ces jeunes utilisent des stratégies différentes pour construire leur projet qui sans elles ne pourrait voir le jour.

\section{Place du Secteur Associatif}

Certains jeunes qui ne bénéficient pas, ou insuffisamment, des apports de l'école ou de l'aide de leur famille, et qui se sentent incapables de réaliser seuls les changements nécessaires à la construction de leur projet, se tournent alors vers des associations de quartier (cf. note 3), ce qui est souvent le cas des jeunes dont il a été question précédemment ${ }^{5}$.

Ces dernières présentent des caractéristiques autres que celles des dispositifs éducatifs classiques. Leur fréquentation est en effet basée sur le volontariat et d'une durée variable (de l'ordre de l'éphémère à quelques années), n’imposant généralement pas de présence régulière. En outre, les relations entre adhérents et avec le personnel sont basées sur une certaine liberté des échanges.

Nous faisons l'hypothèse que ces associations (p. e. les centres sociaux) constituent un espace de créativité qui possède les caractéristiques propres à l'analyse transitionnelle. On peut en effet considérer, à partir des travaux de KAËS (1979), qu'elles constituent une «zone intermédiaire d'expérience » (entre plusieurs cultures), et « quelles favorisent un processus de passage (de transition) entre deux états subjectifs, constituant une zone dans laquelle se joue la question du code culturel » (KAËS, 1979, p. 60).

Dans ces structures plus souples que celles des instances sociales de base telle lécole, les jeunes sont placés sous le regard d'animateurs (le plus souvent de jeunes adultes issus eux aussi d'autres cultures) qui peuvent être considérés comme occupant une fonction particulière pour le jeune dans la mesure où cette relation arrive à un moment opportun pour lui, c'est-à-dire en adéquation avec son cheminement personnel, modifiant ainsi sa relation à son entourage et à lui-même dans le sens d'une sécurité intérieure renforcée. On 
peut considérer que les animateurs occupent alors une fonction de «tiers éducatif ». En outre, la présence d'autres adolescents issus d'autres cultures, multiplie non seulement les possibilités de rencontres, d'échanges, de mises en relation, mais elle offre par là-même, une diversité de codes culturels, ce qui est propice à la créativité.

Or, en raison le plus souvent de représentations erronées de certains habitants du quartier où se trouve le centre social (« il (le centre social) ne sert à rien »; « on y fait n’importe quoi »; « les filles y sont en danger »), certains jeunes ne peuvent les fréquenter régulièrement, voire tout simplement y entrer, tout en le souhaitant car leurs parents s'y opposent. En dépit de cette interdiction, une partie de ces jeunes, sans doute plus motivés que d’autres, et bénéficiant de la connivence de certains animateurs ou encore de laide de camarades qui ont déjà accès, fréquentent cependant le centre social de façon informelle et ponctuelle. D’autres encore, parfois les mêmes, conseillés et soutenus par un animateur du centre qu'ils ont choisi, déploient alors une stratégie qui implique la présence de leur père qu'ils ont convaincu, sans doute avec l'aide des conseils de lanimateur, de rencontrer ce dernier. Il s'agit donc d'un père suffisamment ouvert sur l'extérieur et qui, ne pouvant répondre aux demandes de son fils quant à son projet, mais en en comprenant l'importance pour son enfant, délègue en quelque sorte une partie de son autorité de père à l'animateur en lui « confiant » son fils afin de laider dans la réalisation de son projet (RIARD, 2011). Dans ces circonstances, l'association occupe une fonction d'espace intermédiaire, capable donc d'articuler, de réduire ou de faire accepter l'impact des différences existant entre le père et le fils, permettant de concilier les termes de la contradiction qu'ils traversent tous deux. Cet espace social, c'est une hypothèse forte, vient combler un manque par rapport à un projet que la situation du jeune ne pouvait lui permettre de réaliser sans cet étayage, facilitant ainsi la transition d’un code à un autre sur la base d'un rapprochement entre les deux cultures. Elle permet au jeune dengager plus avant un travail d'affiliation à la culture du pays dans lequel il vit, ou à certaines de ses parties et, au père, de l'accepter, ce qui réduit ainsi le risque que laffiliation du jeune à certaines parties de la culture du pays d’accueil ne se joue contre sa propre filiation; en quelque sorte, contre l'histoire de ses ancêtres. Lassociation constitue de ce fait un « sas » (KAËS, 1979), espace qui n’appartient ni à une culture ni à une autre, mais qui permet au jeune, par les appuis procurés par chaque culture, de se redisposer, de trouver de nouveaux étayages. Lanimateur qui est en quelque sorte le représentant de lassociation, occupe alors une position d'intermédiaire en permettant au père de faire face aux éventuelles remises en question des matériaux de son propre héritage culturel qu’implique cette « délégation ».

Dans ces conditions, le centre social apparait comme une troisième voie à côté de lécole et de la famille, comme ce qui permet lencodage de nouvelles pratiques, jouant le rôle de traitement, de transformation, et ultimement, d’acceptation des différences, en permettant l’encodage de nouvelles pratiques, ici celles liées au projet. Il vient combler un sentiment de manque par rapport à celui-ci, ce que la situation du sujet ne pouvait permettre de réaliser en son absence.

\section{Conclusion}

Au terme d'une présentation et d'une analyse qui ne peuvent être que réduites de ces trois éléments considérés comme « repères » de la construction du projet et retenus pour cet article, il ressort la présence d'une complexité beaucoup plus grande que les pratiques courantes proposées aux adolescents dans le cadre de lorientation scolaire et professionnelle au collège (test de niveau, aptitudes telles que présentée en première partie), ne le laissent généralement entendre dans la construction de leur projet, et il apparaît que cette construction ne peut reposer sur les seules dimensions cognitives et intellectuelles. Le maintien de cette position peut avoir comme effet d'introduire un clivage au sein des jeunes eux-mêmes, risquant d’en conduire certains à construire leur projet en fonction du désir des autres, et, dans tous les cas, de générer une insatisfaction profonde : celle pour les jeunes de découvrir un jour, lorsqu'ils sont déjà engagés dans la vie adulte, que leur projet n’est en quelque sorte pas leur projet, que leur activité 
professionnelle ne correspond pas à de véritables choix, et quen dépit des apparences, leur vie privée n’a pas été nécessairement choisie mais est plutôt le fait des autres. Si la dimension affective ne doit pas non plus primer, elle ne peut cependant être grandement négligée ou occultée, ce que confirment bon nombre de thérapies adultes.

\section{RÉFÉRENCES}

BLOS, P. Les adolescents: Essai de psychanalyse. Paris: Stock, 1967.

CASTELLAN, Y. Adolescence, rupture et continuité. Carrefours de l'Education, v. 24, p. 21-35, 2007. https://doi. org/10.3917/cdle.024.0021

CASTELLAN, Y.; RIARD, E. H. Les 12-17 ans: Le projet de vie et ses voies. Carrefours de l'Education, v. 19, p. 139164, 2005. https://doi.org/10.3917/cdle.019.0139

CHABERT, C. Modalités du fonctionnement psychique des adolescents à travers le Rorschach et le TAT. Psychologie Française, v. 28, n. 2, p. 187-194, 1993.

CICCONE, A. Empiètement imagoïque et fantasme de transmission. In: EIGUER A. (org.). Le générationnel, approche en thérapie familiale psychanalytique. Paris: Dunod, 1997, p. 153-185. (Collection Inconscient et Culture).

CRITES, J. O. Parental identification in relation to vocational interest development. Journal of Educational Psychology, v. 53, n. 6, p. 262-270, 1962. https://doi.org/10.1037/h0040197

ERIKSON, E-H. Adolescence et crise: La quête de l'identité. Paris: Flammarion, 1968.

GINZBERG, E.; GINSBURG, S.; AXERALD, S.; HERMA, C. Occupationnal choice. New York: Columbia University Press, 1951.

GOLSE, B. Le développement affectif et intellectuel de l’enfant. 4. ed. Issy-les-Moulineaux: Masson, 2008.

GUICHARD, J. L’école et les représentations d’avenir des étudiants. Paris: Presses Universitaires de France, 1993.

GUTTON, P. Du changement à la puberté. Adolescence, v. 1, n. 1, p. 7-11, 1983.

HOANG, G. T. Construction du projet de vie chez les adolescents âgés de 15 à 17 ans scolarisés à Hanoi, Vietnam. 2012. Thèse. (Doctorat en Psychologie Sociale Clinique) - Faculté de Philosophie, Université de Picardie Jules Verne, Amiens, 2012.

HUTEAU, M. La psychologie du projet. ADAPT, n. 3, p. 7-13, 1993.

JEAMMET, P. Du familier à létranger. Territoires et trajets de l'adolescent. Neuropsychiatrie de l'Enfance, v. 31, n. 8-9, p. 361-381, 1983. 
KAËS, R. Introduction à lanalyse transitionnelle. In KAËS (org.). Crise, rupture et dépassement. Paris: Dunod, 1979 , p. 1-81.

KAËS, R. Filiation et affiliation. Gruppo, v. 1, n. 1, p. 23-45, 1985.

KAËS, R. L'idéologie, l'idéal, l’idée, l’idole. Paris: Dunod, 2016.

KESTEMBERG, E. Lidentité et l'identification chez les adolescents. Problèmes théoriques et techniques. Psychiatrie de l'Enfant, v. 5, n. 2, p. 441-522, 1962. https://doi.org/10.3917/dunod.chagn.2012.02.0323

LESOURD, S. La construction adolescente. Toulouse: Eres, 2005.

MAHLER, M. Psychose infantile. Paris: Petite Bibliothèque Payot, 1982.

MAINE, J. Estime de soi et parrainage chez les jeunes de 14 à 17ans: De la logique d'assistance à la logique d'investissement. Le rôle d'un livret dépargne. 2009. Thèse. (Doctorat en Psychologie Sociale Clinique) - Faculté de Philosophie, Université de Picardie Jules Verne, Amiens, 2009.

MÂLE, P. La crise juvénile. Paris: Payot, 1962.

MARCELLI, D.; BRACONNIER, A. Adolescence et psychopathologie. 6. ed.. Issy-les-Moulineaux: Masson, 2007.

MEDEJEL, M. Quelques questions posées à propos de léloge des grands frères. In: RASSIAL, J. J. (org.). Y-a-t-il une psychopathologie des banlieues? Toulouse: Eres, 1998. https://doi.org/10.3917/eres.rassi.2002.01.0107

MURRAY, H. A. Exploration de la personnalité. Paris: Presses Universitaires de France, 1953.

RIARD, E. H. Risque d’errance à ladolescence: Proposition de l'hypothèse de loscillation polarisée, Pratiques Psychologiques, v. 1, p.13-21,1996.

RIARD, E. H. Les jeunes et leur projet de vie dans les contextes français et marocains, Journal des Psychologues, $\mathrm{n}$. 233, p. 45-49, 2005.

RIARD, E. H. Place des associations dans lémergence du projet de vie des jeunes issus du Maroc résidant en Picardie (approche qualitative). In: MELYANI, M. /Ingénierie du lien social: Développement et associations. Amiens: Licorne, 2006.

RIARD, E. H. Entre deux cultures. Les associations de quartiers comme tiers éducatif pour des jeunes issus de lémigration. In: XYPAS, C.; FABRE, M.; HETIER, R. Le tiers éducatif, une nouvelle relation pédagogique. Figures et fonctions du tiers en éducation et formation. Bruxelles: De Boeck, 2011, p. 137-152. https://doi.org/10.3917/dbu. xypas.2011.01.0137

RIARD, E-H., PEYRE, V. Etude des facteurs de risque de marginalisation d'une population de 12 à 16 ans de la Région drouaise dans la dialectique individu-société. (Protection Judiciaire de la Jeunesse, Eure et Loir), (Rapport de recherche). 1997. 
RIARD, E-H.; DACHMI, A. Adolescence et projet de vie (Approche de psychologie clinique et sociale), Publication de la Faculté des Lettres et des Sciences Humaines, Rabat, Essais et études, n. 40, 2004.

RIARD, E-H.; ALAGUI, A. Projet professionnel deetudiants marocains en France issus de la migration: Rôle de la famille et de l'université. In: VATZ LAROUSSI, M.; RIARD, E-H.; GELINAS, C.; JOVELIN, E. (org.). Les défis de la diversité. Paris: L'Harmattan, Espaces interculturels, 2013, p. 301-313

SUPPER, D. E. Travail et loisirs dans une économie en flux. L'orientation Scolaire et Professionnelle, v. 17, n. 1, p. 23-32, 1988.

\section{Notes}

1. Les contrats ont associé le département de l'Eure et Loir, le Ministère de la Justice et la Protection Judiciaire de la Jeunesse (RIARD ; PEYRE, 1997); ou encore une Mission Locale d'Île-de-France (CASTELLAN; RIARD, 2005 ; CASTELLAN, 2007). Pour certains travaux, ils ont été réalisés dans le cadre d’actions intégrées (Programme Hubert Curien) relevant du Ministère des Affaires Etrangères (RIARD ; DACHMI, 2004 ; RIARD ; ALAGUI, 2013).

2. Ces travaux ont constitué une base importante de la mise au point d’un outil de repérage des premières difficultés des jeunes de 12-14 ans (non présenté ni pris en compte ici ; -Projet Régional Structurant, Picardie-), et réalisé sur un échantillon représentatif de 2300 sujets, pour ce qui concerne les 57 questions relatives aux dimensions psychosociale et psychoaffective de lépreuve. (Cet outil inclut également les dimensions psychomotrice, cognitive et intellectuelle).

3. Consigne donnée en début de passation concernant ces cartes : « Imaginez une histoire en disant ce qui sest passé avant, ce qui se passe maintenant, et comment l'histoire va se terminer. Pour chacun des personnages de l'histoire, vous direz également, ce qu'il pense et ce qu'il ressent ». Les cartes retenues ont été présentées dans cet ordre : carte 2 (couple parental) pour tous les sujets ; carte 6 (relations mère/fils dans un contexte de malaise); carte mère/filles conçue (et validée) sur le modèle de la carte destinée aux garçons ; carte 7BM : relations père / garçons; relation père / filles conçue et validée sur le modèle de la carte destinée aux garçons ; carte 4 (relations aux pairs) pour filles et garçons. Enfin, présentation de la carte 16 (destinée aux deux sexes.

4. Ce que l’on entend ici par « secteur associatif » recouvre l'ensemble des associations proposées aux jeunes (maisons de quartier, maisons des jeunes et de la culture, centres sociaux, mission locale...) qui les fréquentent sur la base du volontariat. Les activités proposées peuvent être uniques (p. e. soutien scolaire) ou très diversifiées : par exemple outre du soutien scolaire des activités culturelles et sportives. Leur encadrement est assuré par des adultes (animateurs) professionnels ou bénévoles. Leur mode de financement est variable (État, municipalité, région etc..).

5. Nous ne prenons pas en compte dans la suite de notre propos les jeunes qui se rendent dans des associations exclusivement dédiées au « soutien scolaire », car leur fréquentation est généralement contrainte par leurs parents (RIARD, 2006).

Reçu: 01 Mars 2019

Accepté: 17 Sep 2019

Comité éditorial Cedes/Coordination de ce numéro:

Izabel Galvão e Maria Rosa Camargo 